\title{
Chancen für den Klimaschutz durch eine veränderte Mitarbeitermobilität
}

\author{
Was bringt Arbeitnehmer/innen, die für den \\ Arbeitsweg vorwiegend das eigene Auto nutzen, \\ zu einem Wechsel auf klimafreundliche Verkehrs- \\ mittel? Ein aktuelles EU-Förderprojekt identi- \\ fiziert wesentliche Treiber der Wechselbereitschaft \\ anhand eines neuen Ansatzes zur Ermittlung \\ von Mobilitätspräferenzen. Von Philipp Tachkov, \\ Svenja Richler und Arabella Gregor
}

\begin{abstract}
$\mathrm{n}$ Deutschland und in der Europäischen Union sind unter anderem durch das Kyoto-Protokoll, den EU-Rahmen für die Klima- und Energiepolitik bis 2030, das Aktionsprogramm Klimaschutz 2020 und den Klimaschutzplan 2050 wichtige Grundsteine für eine Reduktion von Treibhausgas-Emissionen gelegt worden (United Nations 1998; Europäische Kommission 2014; BMUB 2014; BMUB 2016). Die individuelle Mobilität ist in diesem Zusammenhang ein wichtiges Handlungsfeld, da sie neben den Emissionen aus Gütertransport, Energiewirtschaft, Industrie und sonstiger Brennstoffnutzung einen wesentlichen Anteil aller $\mathrm{CO}_{2}$-Emissionen verursacht (Umweltbundesamt 2017).
\end{abstract}

\section{Beruflich bedingte Mobilität}

Gerade die berufsbedingte Mobilität ist in Ballungsräumen für einen enormen Anteil der Emissionen verantwortlich (Umweltbundesamt 2012). Das am meisten bevorzugte Verkehrsmittel für berufliche Zwecke ist der eigene Pkw, der als Alleinfahrender genutzt wird (motorisierter Individualverkehr, MIV). Mit rund $80 \%$ machte der motorisierte Individualverkehr in 2012 im Vergleich zu öffentlichen Verkehrsmitteln (15,5\%) und der Radmobilität (2,4\%) den deutlich größten Anteil im bundesweiten Berufsverkehr aus (Umweltbundesamt 2012).

Um diese Verteilung der Verkehrsleistung auf die einzelnen Verkehrsmittel zukünftig zugunsten der klimafreundlichen Mobilitätsalternativen zu verändern, werden innovative und attraktive Konzepte benötigt. Aktuell werden diesbezüglich europaweit zahlreiche Programme und Konzepte entwickelt und zur Anwendung gebracht werden. Ob mit innovativen Ansätzen wie der Entwicklung eines Onlinespiels für Mitarbeitende, das zur Nutzung von umweltfreundlichen Alternativen motivieren soll (EU-Projekt MOBI - Promoting Smart Mobility to Employees) oder einem Know-how-Austausch in den Themengebieten Stadtinfrastruktur, öffentlicher Nahverkehr oder Mobilitätsmanagement (EU-Projekt SOLUTIONS Sharing Opportunities for Low Carbon Urban Transportation) viele Initiativen suchen nach funktionierenden Möglichkeiten, die Attraktivität klimafreundlicher Mobilität zu erhöhen. Wichtig sind bei solchen Projekten häufig Ansätze zur Änderung des Verkehrsverhaltens (DENA 2010), zum Beispiel im Rahmen von Sustainable Urban Mobility Plans (Europäische Kommission 2014). Hier stehen das Nutzerverhalten und seine Beeinflussung im Fokus der Betrachtung, so unter anderem in den EU-Projekten CIVITAS 2020 - City VITAlity and Sustainability und Urban Electric Mobility Initiative (UEMI).

Diese Ausrichtung bildet auch die Grundlage für das durch die Europäische Union aus dem Europäischen Fonds für regionale Entwicklung (EFRE) geförderte Projekt Klimafreundliche Mitarbeitermobilität, das von Oktober 2017 bis September 2019 am Institut für Management und Innovation (IMI) der Hochschule Ludwigshafen am Rhein durchgeführt wird. Das Projekt profitiert dabei von der Zusammenarbeit mit wichtigen Partnern wie der Stadt Ludwigshafen am Rhein, Rhein-Pfalz-Kreis, BASF, AbbVie, Technische Werke Ludwigshafen, Sparkasse Vorderpfalz sowie weiteren strategischen Partnern wie der Metropolregion Rhein-Neckar (M-R-N), des Verkehrsverbunds Rhein-Neckar (VRN), der Energieagentur Rheinland-Pfalz, der DB Regio AG und der Rhein-Neckar-Verkehr GmbH (RNV). In dem Projekt werden am Pilotstandort Ludwigshafen Nutzerpräferenzen für die Gestaltung klimafreundlicher Verkehrsmittel auf eine in diesem Bereich neuartige Weise ermittelt und die Ergebnisse zu Empfehlungen für zukünftige Maßnahmen verdichtet. Die hierzu notwendigen Daten werden durch computergestützte Mitarbeiter/innenbefragungen erhoben, deren Kern ein Experiment zur Ermittlung von Nutzer/innenpräferenzen ist. Die gewonnenen Daten erlauben Simulationen, wie stark unterschiedliche Maßnahmen zur Förderung klimafreundlicher Mobilitätsalternativen die Wahrscheinlichkeit, statt des eigenen Autos ein anderes Verkehrsmittel zu verwenden, beeinflussen. Die dadurch gewonnenen Erkenntnisse fließen in die Planungen von beteiligten Gebietskörperschaften und Unternehmen ein. Durch die Beteiligung großer Arbeitgeber an dem Projekt entsteht eine breite und detaillierte Datenbasis, sodass Analyseresultate prinzipiell bezüglich ähnlicher Ballungsräume verallgemeinerbar sind.

In diesem Beitrag stellen wir dar, welche für die Weiterentwicklung klimafreundlicher Mobilität nützlichen Erkenntnisse durch den Ansatz des Projekts gewonnen werden können und illustrieren Anwendungsfälle mit ausgewählten Beispielen. 


\section{Die Determinanten der Verkehrsmittelwahl}

Die Verkehrsmittelwahl eines Individuums ist grundsätzlich von einer Vielzahl von Determinanten abhängig. Neben der grundlegenden Art und Ausgestaltung des Mobilitätsangebots besteht die Frage, welche weiteren infrastrukturellen Lösungen geboten werden, zum Beispiel sichere Abstellanlagen an Bahn-Haltepunkten, um die intermodale Verkehrsmittelnutzung zu fördern. Auch unterstützende Faktoren wie Services (z. B. vereinfachte Buchungsmöglichkeiten) und Nutzungsanreize verschiedener Art (z. B. Radmeilenprogramm) können die Attraktivität und wahrgenommene Wertigkeit einer Mobilitätsalternative erhöhen. Schließlich sind natürlich auch Aufwandsfaktoren wie die durch Verkehrsmittel entstehenden Kosten und Reisedauern von Bedeutung. Alle diese Faktoren und die jeweils dahinterliegenden vielfältigen möglichen Ausprägungen sind grundsätzlich durch Maßnahmen, sei es durch zuständige Gebietskörperschaften, Verkehrsbetriebe oder private Arbeitgeber, zu beeinflussen und spannen insgesamt einen Raum von Handlungsmöglichkeiten für beteiligte Akteure auf.

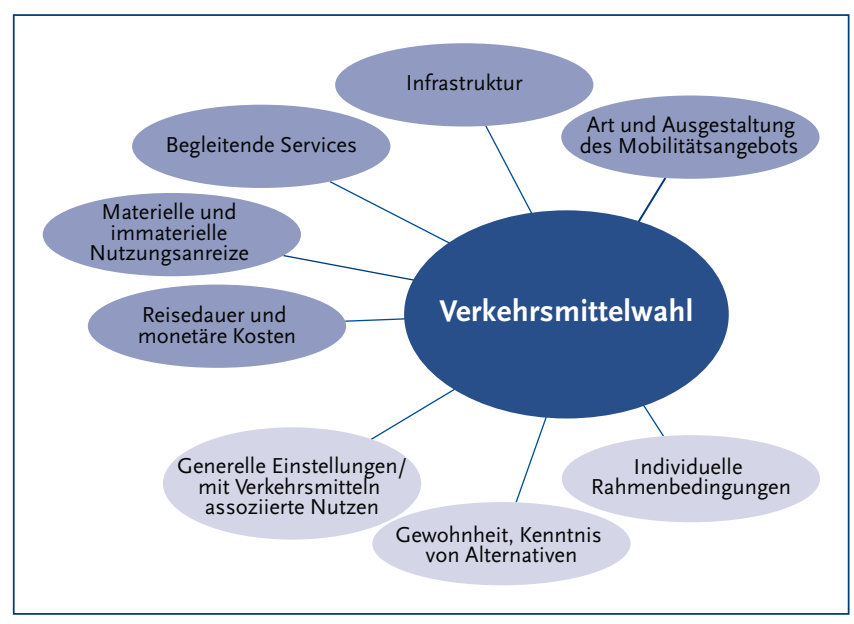

Abbildung 1: Determinanten der Verkehrsmittelwahl

Weiterhin gibt es Einflussfaktoren, die an die individuelle Person und ihre Prägungen gekoppelt und wenig beeinflussbar sind. Hierzu gehören mobilitätsbezogene Einstellungen, Verhaltensgewohnheiten und persönliche Merkmale wie beispielsweise körperliche Beeinträchtigungen, die die Verkehrsmittelwahl von vornherein einschränken. In Summe bleiben jedoch viele Möglichkeiten, wie Rahmenbedingungen gezielt verändert werden können, um die Attraktivität bestimmter Verkehrsmittel zu steigern.

Hinter jedem der gut beeinflussbaren Einflussfaktoren der Verkehrsmittelwahl steht eine große Anzahl von konkreten Gestaltungsoptionen. Nur welche sollen verwirklicht werden? Gerade auch aufgrund der mit der Umsetzung von Maßnahmen stets verbundenen mehr oder minder großen Aufwendungen ist eine zuverlässige Abschätzung über die erzielbaren Effekte von großer Bedeutung. Doch woher können die benötigten Informationen zu den durch die Maßnahmen erzielbaren Effekten kommen und welche Anforderungen sind an sie zu stellen?

\section{Fragestellungen und Methodik}

Woher können wir wissen, welche neuen Maßnahmen die Entscheidung von Arbeitnehmer/innen für klimafreundliche Verkehrsmittel signifikant fördern (und daher umgesetzt werden sollten)? Um entsprechende Informationen zu ermitteln, stehen verschiedene Instrumente bereit. Eine Möglichkeit ist, auf bestehende „Erfahrungswerte“ zurückzugreifen. Dabei ist aber immer eine eingeschränkte Übertragbarkeit auf den aktuellen konkreten Anwendungsfall in Kauf zu nehmen. Auch sind Erfahrungswerte bei gänzlich neuen Ideen häufig gar nicht vorhanden. Eine weitere Option besteht darin, sogenannte „Reallabore“, in denen eine neue Technologie in Kooperation mit der Öffentlichkeit eingebettet in die reale Umgebung getestet wird, einzusetzen. Reallabore sind jedoch aufwendig und teuer und daher nur für wenige Maßnahmen durchführbar. Eine gänzlich andere Herangehensweise besteht im Sammeln von Hinweisen aus dem betrieblichen Vorschlagswesen oder Ideenmanagement. Diese Informationen basieren aber in der Regel auf einer geringen und unsystematischen Datenbasis, weswegen die Relevanz auf diese Weise generierter Vorschläge für die gesamte Mitarbeiterschaft schwer einzuschätzen ist. Schließlich bleibt die Methode der Mitarbeitendenbefragung. Diese ermöglicht kostengünstige, schnelle, und bei entsprechender methodischer Ausführung auch hinreichend verallgemeinerbare Ergebnisse. Jedoch stellt sich bei dieser Methode die Frage nach der Validität der erzielten Ergebnisse, also inwiefern die Resultate auch tatsächlich zuverlässig über den Gegenstand der Befragung, nämlich den Nutzen durch Maßnahmen für verbesserte Mobilität, Auskunft geben können. Bei klassischen Befragungsformen gibt es in diesem Zusammenhang einige kritische Aspekte zu beachten. Bei typischen Abfragen gewünschter Angebote und Anreize zeigt sich häufig eine starke Tendenz zu einem generell hohen Zustimmungsanteil („Wunschkonzert“). Im Allgemeinen ist die Beurteilung von Einzelaspekten (z. B. verbesserte Abstellanalagen für Radfahrer am Arbeitsort) schwierig, da sie aus der gesamten Entscheidungssituation „Nehme ich das Auto oder Rad, ÖPNV oder eine intermodale Möglichkeit“ herausgelöst ist und damit wenig Realitätsbezug aufweist. Es fehlen der Bezug zu anderen relevanten Merkmalen und der Vergleich mit anderen möglichen Verkehrsmitteln und ihren jeweiligen Ausgestaltungen (verbesserte Radabstellanlagen werden insbesondere dann interessant, wenn für Radmobilität auch sonst gute Bedingungen herrschen und andere Verkehrsmittel wenig attraktiv sind).

Welche Anforderungen sollte aber eine Methodik zur Erfassung der Nutzerbedürfnisse für Mobilitätslösungen erfüllen? Zunächst sollte es möglich sein, anhand erhobener Daten be- 
stimmen zu können, wie stark der Einfluss einzelner Fördermaßnahmen klimafreundlicher Mobilität auf die generelle Entscheidung ist, ein klimafreundliches Verkehrsmittel zu nutzen. Weiterhin sollten Aussagen darüber möglich sein, ob es diesbezüglich starke Unterschiede zwischen verschiedenen Maßnahmen gibt. Ein weiteres Kriterium ist, dass die Nutzenzusammenhänge zwischen verschiedenen Maßnahmen quantifiziert werden können. Bei einer „Infrastrukturmaßnahme Radschnellweg" erscheint plausibel, dass der Nutzen von guten Radabstellanlagen sowie am Arbeitsort zur Verfügung stehenden Umkleiden und Duschen ansteigt, wenn hochwertigere Fahrräder verwendet werden und größere Strecken zurückgelegt werden. Beides wäre im Fall eines neuen Radschnellweges sicherlich der Fall. Letztlich sollten auf Basis erhobener Daten Aussagen darüber getroffen werden können, was stimmige „Maßnahmenbündel“ sind. Hier geht es um die Identifikation mehrerer Maßnahmen, die sich gegenseitig verstärken und somit sinnvolle Kombinationen darstellen.

\section{Untersuchung von Mobilitätsbedürfnissen}

Ausgehend von den voranstehenden Überlegungen wird zur Gewinnung valider Informationen zu Mobilitätsbedürfnissen eine Methodik benötigt, die die skizzierten Anforderungen erfüllt. Als am besten geeignet erscheinen Discrete-Choice-Experiments, eine Methodik zur Präferenzmessung, die eingebettet in eine Onlinebefragung durchgeführt werden kann und die im EU-Förderprojekt Klimafreundliche Mitarbeitermobilität zur Anwendung kommt. Die Methodik ist in den Sozialwissenschaften wie auch in der praktischen Anwendung weithin etabliert, um in einer frühen Phase der Angebotsentwicklung valide Informationen zu den Präferenzen von Individuen bezüglich verschiedener denkbarere Angebotsalternativen zu erhalten. Da bei der Mobilitätsentscheidung auch die Annahme zugrunde gelegt werden kann, dass es sich hier - trotz des stark habitualisierten Verhaltens - in vielen Fällen um eine Entscheidung zwischen Angebotsalternativen handelt, ist der Transfer der Methodik geeignet, um mehr über das Nutzerverhalten bei hypothetischen Mobilitätsangeboten herauszufinden.

Discrete-Choice-Experimente basieren auf der theoretisch fundierten Annahme, dass Personen in Entscheidungssituationen den möglichen Handlungsalternativen intuitiv einen Gesamtnutzen zuordnen und sodann diejenige Alternative gewählt wird, die den höchsten Nutzen verspricht (Telser 2002). Der Nutzen der Alternativen hängt dabei von den konkreten Ausprägungen der Merkmale ab, mit denen die Alternativen beschrieben sind (im Fall von Mobilitätsangeboten beispielsweise die Verbindungen, Taktfrequenz und Informationsdienstleistungen rund um öffentliche Verkehrsmittel). Aus den Angaben von Testpersonen, welche Alternative sie gesamthaft präferieren, kann der Beitrag einzelner Merkmalsausprägungen auf den Gesamtnutzen ermittelt werden. Diese Nutzenwerte können schließlich dazu verwendet werden, die Präferenz der Nutzer/innen für aus verschiedenen Merkmalen zusammengesetzte Mobilitätsangebote zu simulieren. Mit Discrete-Choice-Experimenten können die Einflüsse auf die Auswahl aus hypothetischen Handlungsalternativen realitätsnah ermittelt werden, damit geht eine generell hohe Datenqualität einher (Orme 2010).

\section{Empirisches Studiendesign}

Die zentrale Fragestellung des Projekts, wie klimafreundliche Mobilitätsangebote aus der Nutzerperspektive mit passenden Maßnahmen weiterentwickelt werden sollten, hat vor allem eine Verhaltensänderung der gewohnheitsmäßigen Allein-Autofahrer (motorisierter Individualverkehr) im Blick. Im Rahmen eines Discrete-Choice-Designs sind daher zunächst die möglichen Mobilitätsalternativen dieser Zielgruppe zu bestimmen. Im Projekt Klimafreundliche Mitarbeitermobilität findet hierzu eine intensive Zusammenarbeit aller Projektpartner statt, um nur relevante Merkmale aufzunehmen, insbesondere solche, die den jeweiligen Rahmenbedingungen und infrastrukturellen Möglichkeiten gerecht werden.

Neben der gewohnten Alternative „Pkw-Alleinfahrer“ wurden die grundständigen Mobilitätsoptionen „Fahrrad, Pedelec, E-Bike“, „Öffentliche Verkehrsmittel“ und „Pkw-Fahrgemeinschaft" definiert, wobei auch intermodale Verknüpfungsmöglichkeiten berücksichtigt sind. Weiterhin wurden wichtige Merkmale bestimmt, die das Angebot der jeweiligen Mobilitätsoption beschreiben (Abbildung 2).

So kann die Alternative „Pkw-Alleinfahrer“ beispielsweise mittels der Verkehrssituation (mögliche Ausprägungen sind z. B. „freie Fahrt" sowie Verkehrsbehinderungen und Stauszenarien) und auch der Parksituation beschrieben werden. Analog sind für die alternativen Mobilitätsoptionen verschiedene Merkmale und je Merkmal eine Reihe von Gestaltungsoptionen denkbar. Bei der Mobilitätsoption „Fahrrad, Pedelec, E-Bike“ können im Merkmal „Art des vorherrschenden Radwegs" zum Beispiel verschiedene Levels von Radwegen - vom Radschutzstreifen auf der Fahrbahn über den abgetrennten Radweg bis hin zu einem modernen Radschnellweg - berücksichtigt werden. In Summe definieren die berücksichtigten Mobilitätsoptionen samt den zugeordneten Merkmalen und den einzelnen Merkmalsausprägungen die Stimuli des Discrete-Choice-Experiments.

Abbildung 3 illustriert, wie das Experiment im Rahmen einer Erhebung unter gewohnheitsmäßigen Autopendlern umgesetzt werden kann. Abgebildet ist eine typische Entscheidungssituation, in der die vier Mobilitätsoptionen in einem Szenario, in dem es um die Frage der Verkehrsmittelwahl für den Arbeitsweg geht, zur Auswahl stehen. Jede Mobilitätsoption ist mit konkreten Ausprägungen unterschiedlicher Merkmale beschrieben, im Fall der klimafreundlichen Alternativen auch mit unterschiedlichen Maßnahmen zur Steigerung ihrer Attraktivität. Aufgabe der Teilnehmer/innen ist es, die Mobilitätsoptionen $\mathrm{zu}$ vergleichen und diejenige $\mathrm{zu}$ benennen, die unter den beschriebenen Umständen als die attraktivste be- 


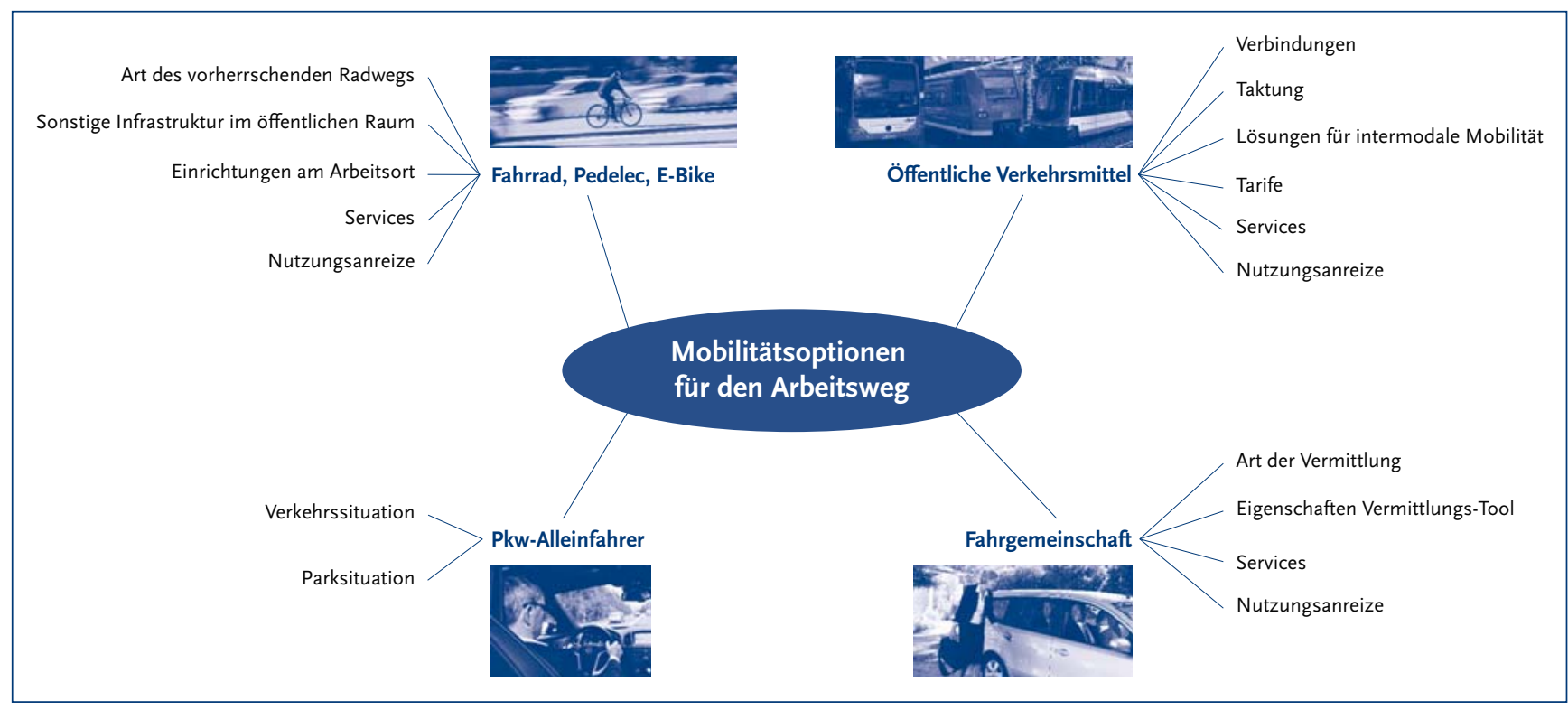

Abbildung 2: Mobilitätsoptionen und Beispiele für beschreibende Merkmale

trachtet wird. Indem viele Teilnehmer/innen jeweils mehrere, nach einem Algorithmus zusammengestellte Szenarien beantworten, entsteht eine große und detaillierte Datenmenge zum Einflussgewicht einzelner Maßnahmen auf die Wahrscheinlichkeit, eine klimafreundliche Mobilitätsalternative zu bevorzugen. Im Fall der Mobilitätsoption „Pkw-Alleinfahrer“ können so zum Beispiel die Einflüsse von „Push-Faktoren“ - also Faktoren, die die Attraktivität des motorisierten Individualverkehrs herabsetzen - getestet werden. Im Fall der klimafreundlichen Mobilitätsalternativen sind es hingegen die Wirkungen von „Pull-Faktoren“- das heißt Faktoren, die die Attraktivität dieser Alternativen steigern. Anders als in herkömmlichen Abfragen müssen sich die berücksichtigten Maßnahmen in einem solchen experimentellen Setting dem "Wettbewerb“ mit anderen Angeboten und Einflussgrößen stellen. Die Angabe der Präferenz für eine Mobilitätsoption hängt also von dem Abwägen diverser (je nach Individuum unterschiedlich relevanter) Aspekte ab. Dadurch wird die „relative Stärke“ von Maßnahmen bestimmt, die Folge sind deutlich belastbarere Daten zu deren wahrgenommener Attraktivität.

\section{Beispiele möglicher Erkenntnisse}

Im Fall von Push-Faktoren kann anhand der im Experiment gewonnenen Daten simuliert werden, wie sich die Verkehrsmittel-Präferenz der untersuchten Stichprobe allein aufgrund des Auftretens von Stauszenarien verändert. Abbildung 4 zeigt in einer Simulation mit drei berücksichtigten Verkehrsmitteln anhand der Daten eines Projekt-Untersuchungsstandorts, wie sich die Präferenz von gewohnheitsmäßigen Autopendler/innen in Abhängigkeit der Verkehrssituation auf klimafreundliche Mobilitätsalternativen wie Radmobilität und öffentliche Verkehrsmittel verschiebt. Dabei können auch „Akzeptanzbe- reiche" ermittelt werden, in denen die sinkende Attraktivität des motorisierten Individualverkehrs aufgrund von Verkehrsbehinderungen noch nicht in großem Ausmaß zu einer stärkeren Präferenz für Alternativen führt. Spiegelbildlich sind Ereignisse (z. B. Staus von bestimmter Dauer) identifizierbar, die die Attraktivität des MIV in der Wahrnehmung des Nutzers so weit sinken lassen, sodass der größte Gesamtnutzen durch ein alternatives klimafreundliches Verkehrsmittel erreicht wird.

Eine im Zusammenhang mit einer Förderung klimafreundlicher Arbeitswegmobilität zentrale Fragestellung ist, wie stark unterschiedliche Maßnahmen und Angebote auf die Präferenz für klimafreundliche Verkehrsmittel einwirken (Pull-Faktoren). Mit den durch den experimentellen Befragungsansatz gewonnenen Daten können analog zur Betrachtung der Wirkung von Push-Faktoren Simulationen durchgeführt werden, die zeigen, um welches Ausmaß sich die Präferenzanteile für klimafreundliche Verkehrsmittel steigern, wenn bestimmte Angebote gemacht werden. Damit kann die Effektivität einzelner Maßnahmen direkt verglichen werden. Im Beispiel (Abbildung 5) werden Simulationsergebnisse für einen Projekt-Untersuchungsstandort dargestellt. An der unterschiedlichen Spannweite der Werte je Merkmal zeigt sich die unterschiedliche Bedeutung, die gewohnheitsmäßige Autofahrer/innen diesen Maßnahmen beziehungsweise Angeboten zumessen. Während es einen großen Unterschied für die Zielgruppe macht, welcher Takt bei öffentlichen Verkehrsmitteln angeboten wird, ist die Spannweite der Werte bei der Frage der Anzahl der Umstiege bereits geringer. Maßnahmen, die eine Verknüpfung öffentlicher Verkehrsmittel mit anderen Verkehrsmitteln ermöglichen, steigern im Durchschnitt der zugrunde gelegten Stichprobe zwar auch die Präferenz für öffentliche Verkehrsmittel, haben im Vergleich zu den beiden anderen Merkmalen aber einen geringeren Einfluss. 
Weiterhin können sequenzielle Simulationen der Präferenz für Verkehrsmittel vorgenommen werden, um beispielsweise erwartete Entwicklungen bei Pull- und Push-Faktoren gesamthaft $\mathrm{zu}$ betrachten. So kann in einem ersten Schritt ermittelt werden, wie stark eine geplante Verbesserung des Angebots im Bereich öffentlicher Verkehrsmittel zu einer Verschiebung der Präferenz zur klimafreundlichen Alternative führt. In vielen Städten sind indes teils langwierige Renovierungs- oder Baumaßnahmen bei Hauptverkehrsstraßen oder Brücken geplant, wodurch Verkehrsprobleme entstehen und die Attraktivität des motorisierten Individualverkehrs sinkt. Durch einen weiteren Simulationsschritt, der diese Entwicklungen vorwegnimmt, kann frühzeitig die Steigerung der Präferenz für öffentliche Verkehrsmittel aufgrund solcher Ereignisse abgeschätzt werden. Mögliche entstehende Kapazitätsengpässe bei öffentlichen Verkehrsmitteln können so - in Verbindung mit weiteren Daten - rechtzeitig erkannt und passende Maßnahmen ergriffen werden.

\section{Fazit}

Eine veränderte tägliche Arbeitswegmobilität, die stärker als bisher durch die Nutzung klimafreundlicher Verkehrsmittel geprägt ist, kann einen signifikanten Beitrag zur Einsparung von $\mathrm{CO}_{2}$-Emissionen leisten. Um passende Angebote für einen dauerhaften Umstieg bereitstellen zu können, ist es notwendig, die betreffenden Personen und ihre Bedürfnisse in den Mittelpunkt der Weiterentwicklung klimafreundlicher Mobilitätsoptionen zu stellen, die täglich mit dieser Entscheidung konfrontiert werden. Interdisziplinäre Ansätze können in diesem Zusammenhang neue Erkenntnisse für ein besseres Verständnis des Nutzerverhaltens liefern. Im Einklang mit anderen europaweiten Mobilitätsprojekten, in denen das Nutzerverhalten und seine Determinanten betrachtet werden, liefert das laufende EU-Förderprojekt Klimafreundliche Mitarbeitermobilität spezifische

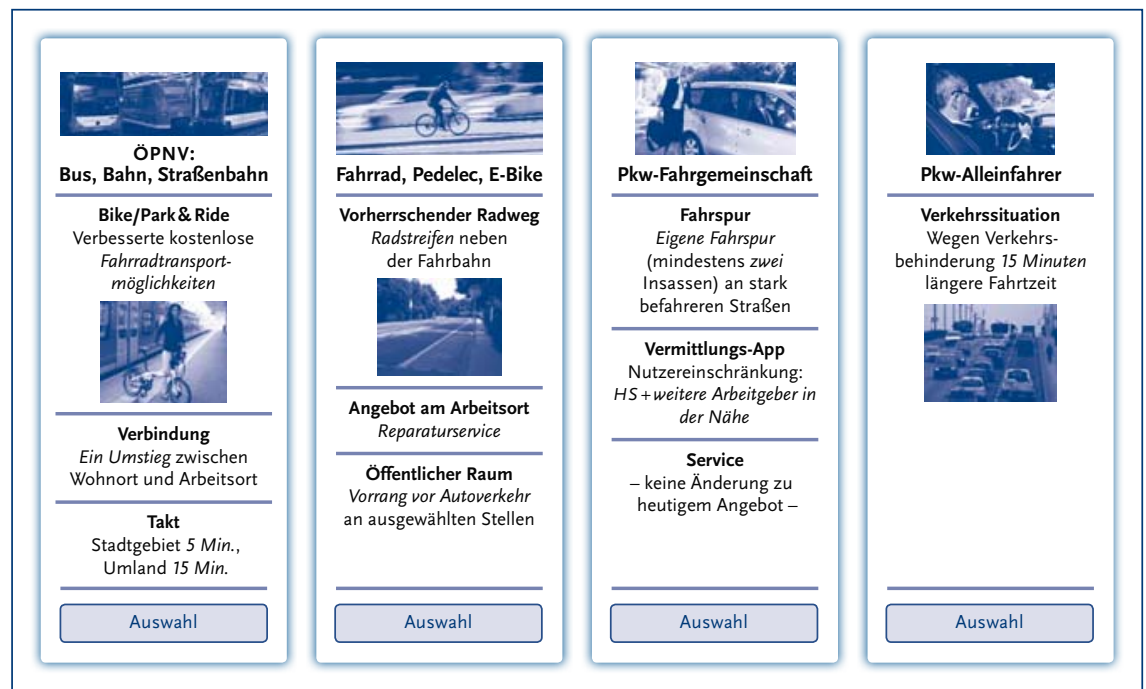

Abbildung 3: Beispiel einer Entscheidungssituation

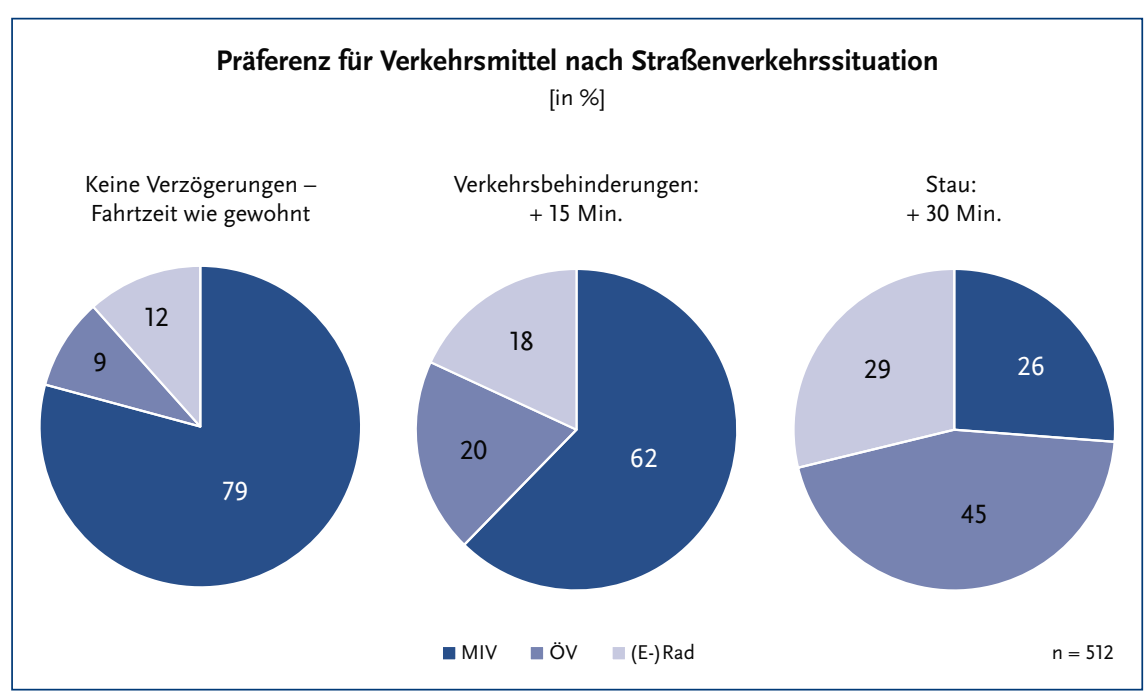

Abbildung 4: Veränderung der Präferenz für Verkehrsmittel durch Verkehrsbehinderungen (Simulation)

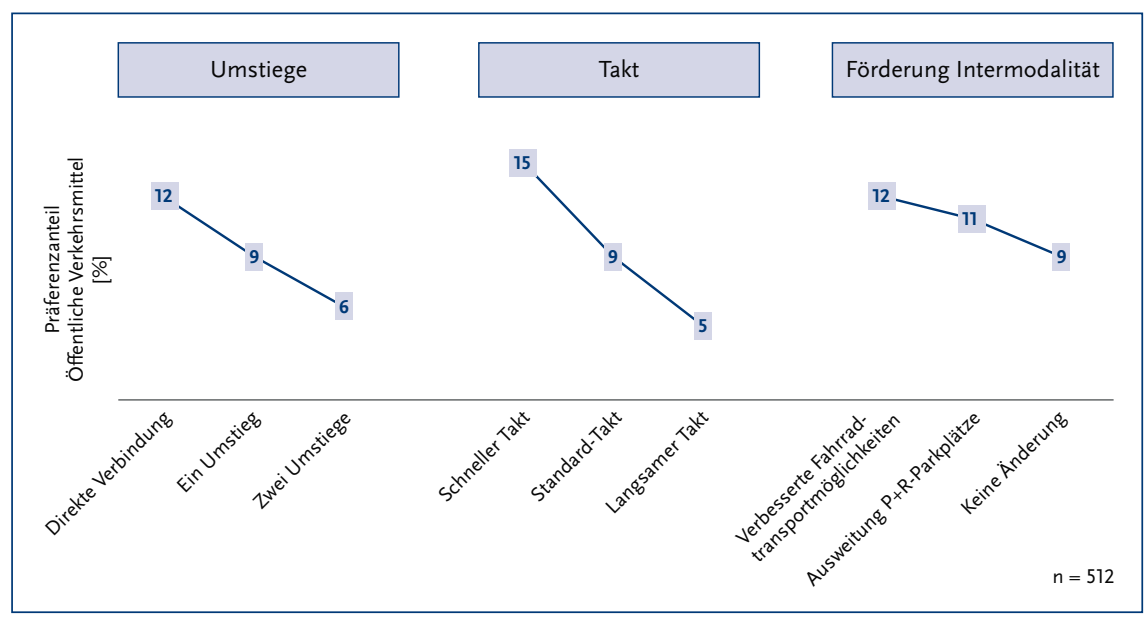

Abbildung 5: Veränderung der Präferenz für öffentliche Verkehrsmittel durch verschiedene Angebote 
Daten, die neuartige Erkenntnisse zu den Effekten verschiedener Angebote auf die Präferenz für ein klimafreundliches Verkehrsmittel ermöglichen. Insbesondere wenn keine belastbaren Vergleichsdaten vorliegen, kann der Ansatz wertvolle Informationen zu der Frage liefern, wie Verkehrsmittelnutzer auf veränderte Angebote und Rahmenbedingungen reagieren. Insbesondere folgende Fragen können mithilfe dieses Ansatzes gezielt erforscht werden:

- Welche Fördermaßnahmen sind die effektivsten, um die Nutzung klimafreundlicher Verkehrsmittel auszuweiten?

- Können einzelne Fördermaßnahmen klimafreundlicher Mobilität die Präferenz der Zielgruppe für das betreffende Verkehrsmittel in signifikanter Weise steigern?

- Welche Maßnahmen bilden in der Wahrnehmung der Nutzer/innen sinnvolle Maßnahmenbündel?

- Gibt es „Kannibalisierungseffekte“ in dem Sinne, dass eine Fördermaßnahme für ein klimafreundliches Verkehrsmittel zur Verringerung der Präferenz für ein anderes klimafreundliches Verkehrsmittel führt, aber kaum zur Reduktion der Präferenz für den motorisierten Individualverkehr?

- In welchen Teilbereichen klimafreundlicher Mobilität besteht das größte Potenzial zur Reduktion des Anteils an motorisiertem Individualverkehr?

Verkehrsplaner/innen und Mobilitätsmanager/innen bei kommunalen Verwaltungen, Verkehrsverbünden und -betrieben und in Unternehmen ist hiermit ein effektives und kostengünstiges Werkzeug an die Hand gegeben, um anstehende Entscheidungen zu Maßnahmen und Investitionen fundierter treffen zu können. Die Gefahr von Fehlentscheidungen aufgrund über- oder unterschätzter Nutzerreaktionen kann auf diese Weise weiter verringert, der Erfolg des Ausbaus klimafreundlicher Mobilität für den Arbeitsweg und darüber hinaus gesteigert werden.

\section{Literatur}

Bundesministerium für Umwelt, Naturschutz und nukleare Sicherheit (BMU) (2014): Aktionsprogramm Klimaschutz 2020.

Bundesministerium für Umwelt, Naturschutz und nukleare Sicherheit (BMU) (2016): Klimaschutzplan 2050 - Klimaschutzpolitische Grundsätze und Ziele der Bundesregierung.

Deutsche Energie-Agentur GmbH (DENA) (2010): effizient mobil. Das Aktionsprogramm für Mobilitätsmanagement, Programmdokumentation 2008-2010.

Europäische Kommission (2014): Rahmen für die Klima- und Energiepolitik bis 2030.

Orme, B. (2010): Getting Started With Conjoint Analysis: Strategies for Product Design And Pricing Research. Madison, Research Publishers.

Telser, H. (2002): Nutzenmessung im Gesundheitswesen, die Methode der Discrete Choice Experimente. Hamburg, Kovac.

Umweltbundesamt (UBA) (2012): Daten zum Verkehr. Ausgabe 2012

Umweltbundesamt (UBA) (2017): Berichterstattung unter der Klimarahmenkonvention der Vereinten Nationen und dem Kyoto-Protokoll 2017. Nationaler Inventarbericht zum Deutschen Treibhausgasinventar 1990-2015.

United Nations (1998): Kyoto Protocol to the United Nations Framework Convention on Climate Change.

AUTOR/INNEN + KONTAKT
Philipp Tachkov ist Projektleiter am
der Hochschule Ludwigshafen.
Institut für Management und Innovation (IMI)
Svenja Richler und Arabella Gregor sind
wissenschaftliche Mitarbeiterinnen am
Institut für Management und Innovation (IMI).
Institut für Management und Innovation (IMI),
Hochschule Ludwigshafen, Ernst-Boehe-Str.4,
67059 Ludwigshafen am Rhein. Tel.: +49 621 5203284,
E-Mail: Philipp.Tachkov@hs-lu.de,
Svenja.Richler@hs-lu.de, Arabella.Gregor@hs-lu.de,
Website: imi.hs-lu.de

\section{P wie Pioniere}

Nachhaltiges Wirtschaften ist möglich - das beweisen zahlreiche engagierte Persönlichkeiten, die das Umwelt- und Nachhaltigkeitsmanagement in Unternehmen, aber auch in Wissenschaft, Medien und anderen Institutionen erfolgreich voranbringen. "Pioniere der Nachhaltigkeit" stellt einige dieser Menschen vor: mal als Vordenker ihrer Disziplin, mal als Vorbild ihrer Branche. Und immer mit außergewöhnlich großem persönlichen Engagement.

Bundesdeutscher Arbeitskreis für Umweltbewusstes Management (B.A.U.M.) e. V. Pioniere der Nachhaltigkeit

25 Jahre B.A.U.M.-Umweltpreis

144 Seiten, Broschur, vierfarbig mit vielen Bildern, 24,- Euro,

ISBN 978-3-96238-075-5

Erhältlich im Buchhandel oder versandkostenfrei innerhalb Deutschlands bestellbar unter www.oekom.de

Die guten Seiten der Zukunft 